\title{
Propriedades tecnológicas de painéis aglomerados produzidos com misturas de fibras e maravalhas de Pinus spp
}

\author{
Technological properties of particleboards made from \\ mixture of fibers and wood shavings from Pinus spp
}

\author{
Alexsandro Bayestorff da Cunha1, Polliana D'Angelo Rios', \\ Cleide Beatriz Bourscheid ${ }^{2}$, Daniella Del Castanhel Kniess' ${ }^{2}$, Franciele Oliveira Córdova², \\ Helena Cristina Vieira ${ }^{2}$ e Vanessa Zulianello da Silveira ${ }^{3}$
}

\begin{abstract}
Resumo
O presente estudo teve como objetivo determinar a viabilidade técnica da produção de painéis aglomerados de misturas de fibras e maravalhas de Pinus spp por meio das propriedades físicas e mecânicas. A matéria-prima utilizada para a produção dos painéis foi composta por fibras retiradas do processo produtivo de MDF, maravalha coletada no processo de aplainamento da madeira, resina uréia-formaldeído e emulsão de parafina. O delineamento experimental envolveu cinco tratamentos homogêneos nas proporções de: 100 partes de fibras para 0 partes de maravalha (T1), 75:25 (T2), 50:50 (T3), 25:75 (T4) e 0:100 (T5). Foram produzidos três painéis por tratamento com $650 \mathrm{~kg} \cdot \mathrm{m}^{-3}$ de massa específica, $12 \%$ de resina, $1 \%$ de emulsão de parafina e ciclo de prensagem de $40 \mathrm{~kg} \mathrm{~cm}^{-2}$ de pressão, $160^{\circ} \mathrm{C}$ de temperatura por um tempo de 8 minutos. A determinação da massa específica, absorção de água, inchamento em espessura e tração perpendicular foram realizados de acordo com os procedimentos da ASTM (2006); o ensaio de flexão estática seguiu os procedimentos da norma DIN (1982) e o ensaio de arrancamento de parafuso as indicações da ABNT (2013). Com a verificação da normalidade dos dados e da homogeneidade das variâncias, foi aplicada a análise da variância e o teste de Scott-Knott a $95 \%$ de probabilidade. Os resultados demonstraram que os valores médios de estabilidade dimensional dos painéis foram regulares em relação às normas de referência. No entanto, o incremento da quantidade de maravalha nos painéis prejudicaram as propriedades mecânicas, principalmente nos valores de módulo de elasticidade à flexão estática e adesão interna.
\end{abstract}

Palavras-chave: Painéis reconstituídos de Pinus spp, resíduos de madeira, fibras.

\begin{abstract}
This study aimed to determine the technical feasibility of the production of particleboard from fibers and wood shaving of Pinus spp through physical and mechanical properties. The raw material used for producing panels was composed of fibers taken of the MDF process, wood shaving collected in the flattening process, urea formaldehyde resin and paraffin emulsion. The experiment involved 5 treatment homogeneous in the proportions: 100 parts of fibers to 0 parts of wood shavings (T1), 75:25 (T2), 50:50 (T3), 25:75 (T4) and 0: 100 (T5). 3 panels were produced by treatment with $650 \mathrm{~kg} \cdot \mathrm{m}^{-3}$ density, $12 \%$ resin, $1 \%$ paraffin emulsion and pressure of $140 \mathrm{~kg} . \mathrm{cm}^{-2}$, temperature of $160 \mathrm{oC}$ for 8 minutes. The determination of specific gravity, water absorption, and thickness swelling, tension perpendicular to surface were performed according to the procedures of ASTM (2006); the static bending test followed the procedures of DIN (1982) and screw withdrawal resistance the indications of ABNT (2013). With the verification of data normality and homogeneity of variance an analysis of variance was carried out and the Scott-Knott test at $95 \%$ probability. The results showed that the average values of dimensional stability of the panels were regular in relation to the reference standards. However, the increased amount of wood shaving in the panels caused a decrease in the mechanical properties, especially the modulus of elasticity in the static bending and in internal adhesion.
\end{abstract}

Keywords: Wood panels, waste of flattening, fibers.

${ }^{1}$ Professor Titular do Departamento de Engenharia Florestal. UDESC - Universidade do Estado de Santa Catarina / Centro Agroveterinário. Av. Luiz de Camões, 2090 - Conta Dinheiro - 88520000 - Lages, SC, Brasil. E-mail: alexsandro.cunha@udesc.br; polliana.rios@udesc.br.

${ }^{2}$ Mestranda em Engenharia Florestal. UDESC - Universidade do Estado de Santa Catarina / Centro Agroveterinário. Av. Luiz de Camões, 2090 - Conta Dinheiro - 88520000 - Lages, SC, Brasil. E-mail: cleidib@gmail.com; danikniess@hotmail. com; foc fran@hotmail.com; lenacristin@hotmail.com.

${ }^{3}$ Engenheira Florestal. UDESC - Universidade do Estado de Santa Catarina / Centro Agroveterinário. Av. Luiz de Camões, 2090 - Conta Dinheiro - 88520000 - Lages, SC, Brasil. E-mail: vanessazulianello@gmail.com.

Sci. For., Piracicaba, v. 44, n. 112, p. 841-849, dez. 2016 DOI: dx.doi.org/10.18671/scifor.v44n112.06 


\section{INTRODUÇÃO}

Painéis reconstituídos de madeira, como os aglomerados, são produzidos a partir de partículas de madeira impregnadas com resina e consolidados por meio da aplicação de calor e pressão (IWAKIRI, 2005). São produtos amplamente empregados, principalmente para fabricação de móveis e consumido em menor escala, na construção civil (BIAZUS et al., 2010).

No Brasil, a produção de painéis aglomerados desde os seus primórdios (1966) até os dias atuais, tem sido pelo processo de formação em multicamadas com partículas de geometria variada, sendo a matéria-prima oriunda de madeira roliça que é transformada em partículas com o uso de picadores. As espécies de madeira utilizadas são geralmente provenientes de reflorestamentos do gênero Pinus e em menor escala do Eucalyptus (BRITO et al., 2004)

As utilizações dos painéis de madeira estão diretamente associadas às propriedades físicas e mecânicas dos mesmos. As principais restrições técnicas para o uso e a aplicação dos diferentes tipos de painéis de madeira, envolvem características como: resistência mecânica, uso interior ou exterior, uniformidade da superfície, tolerância à usinagem, resistência à fixação de parafusos (ABIMCI, 2009). Os painéis de média densidade produzidos com uréia-formaldeído em geral são destinados para uso interno (CAMPOS; LAHR, 2004).

$\mathrm{O}$ atual consumo de madeira em grande escala, pelos diversos setores da sociedade, faz com que surjam discussões e questionamentos sobre os impactos dos resíduos madeireiros ao ecossistema, instigando a ciência florestal no desenvolvimento de pesquisas sobre soluções mitigadoras dos impactos ambientais gerados nos processos produtivos, onde se tem a matéria-prima madeira como principal componente do processo (WEBER, 2011). Assim faz-se necessário buscar novas composições para utilização destes resíduos disponíveis.

De acordo com a ABRAF (2013), as indústrias de base florestal geram cerca de 41 milhões de toneladas de resíduos. Estes podem ser encontrados nas diferentes etapas de processamento ou oriundos da colheita florestal. Deste modo, há uma grande demanda por estudos para viabilizar a utilização destes resíduos, como na formação de painéis aglomerados, ocasionando também um aumento no valor agregado do produto (WEBER, 2011).

Os resíduos das indústrias florestais possuem dimensões variadas, entretanto, podem ser processados nos equipamentos utilizados na indústria, possibilitando a produção de partículas com geometria adequada, sem causar maiores dificuldades durante o processo (HRÁZSKÝ; KRÁL, 2003).

Sendo assim, o objetivo desse estudo foi avaliar a viabilidade técnica da produção de painéis aglomerados com mistura de fibras e maravalhas de Pinus spp por meio da determinação de propriedades físicas e mecânicas de painéis produzidos com resina uréia-formaldeído.

\section{MATERIAL E MÉTODOS}

\section{Matéria - prima}

Foram utilizadas maravalhas e fibras de Pinus spp, sem distinção de espécie, idade e massa específica em função da coleta ter sido realizada diretamente nos processos industriais que não tem o controle supracitado; resina uréia-formaldeído e emulsão de parafina. As maravalhas resultantes do processo de aplainamento foram coletadas na serraria da Madepar Indústria e Comércio de Madeiras LTDA, localizada na cidade de Lages - SC, enquanto que as fibras, a resina e a parafina foram obtidas na empresa Sudati S.A. de Otacílio Costa - SC.

A composição química quantitativa das maravalhas utilizadas para a produção dos painéis foi em média: $14,77 \%$ de extrativos totais; $0,60 \%$ de cinzas; $25,10 \%$ de lignina e o teor de holocelulose foi de 59,47\%. Enquanto que para as fibras fornecidas pela empresa Sudati, Miguel et al. (2016) encontraram valores de 3,04\% para extrativos totais; $0,44 \%$ para cinzas; $31,88 \%$ para lignina e $64,03 \%$ para o teor de holocelulose.

A resina apresentava as seguintes características: $\mathrm{pH}=8,1$, teor de sólidos $=70 \%$ e viscosidade $=$ $250 \mathrm{cp}$; já a emulsão de parafina: $\mathrm{pH}=9,8$, teor de sólidos $=62 \%$ e viscosidade $=500 \mathrm{cp}$.

Não foi realizado nenhum preparo adicional ou classificação granulométrica das partículas de maravalhas e fibras em função do objetivo do estudo, sendo aplicada somente a secagem a $60 \pm 2{ }^{\circ} \mathrm{C}$ até umidade final de $4 \%$. 


\section{Confecção dos painéis}

A aplicação da resina e da emulsão de parafina, foi feita de forma independente, em uma encoladeira. Para tal, foi seguida as proporções industriais, de 10\% de resina e 1\% de emulsão de parafina (base massa seca das partículas). Para cada tratamento foram confeccionados três painéis com massa específica nominal de $650 \mathrm{~kg} \cdot \mathrm{m}^{-3}$ e camadas homogêneas, sendo:

- tratamento $1(\mathrm{~T} 1)=100 \%$ de fibras;

- tratamento 2 (T2) $=75 \%$ de fibras e $25 \%$ de maravalha;

- tratamento 3 (T3) $=50 \%$ de fibras e $50 \%$ de maravalha;

- tratamento $4(\mathrm{~T} 4)=25 \%$ de fibras e $75 \%$ de maravalha;

- tratamento 5 (T5) $=100 \%$ de maravalha.

O colchão de partículas foi submetido a uma pré-prensagem em uma prensa hidráulica com pressão específica de $4 \mathrm{Kgf.cm}{ }^{-2}$, sendo posteriormente direcionado para a prensagem a quente. $\mathrm{O}$ ciclo de prensagem foi de $40 \mathrm{kgf.cm}{ }^{-2}$ de pressão, $160^{\circ} \mathrm{C}$ de temperatura por 8 minutos. Após a prensagem, os painéis foram esquadrejados para dimensões de $40 \times 40 \times 1,55 \mathrm{~cm}$, de onde seguiram para a sala de climatização $\left(20 \pm 3^{\circ} \mathrm{C}\right.$ de temperatura e $65 \pm 5 \%$ de umidade relativa $)$ e posterior retirada dos corpos de prova para realização dos ensaios físico-mecânicos.

\section{Propriedades físico- mecânicas avaliadas e análise estatística}

Os ensaios físicos de massa específica, absorção de água, inchamento em espessura e mecânico de tração perpendicular foram realizados de acordo com os procedimentos da ASTM (2006); a razão de compactação foi calculada com base nos procedimentos descritos por Maloney (1993), o ensaio de flexão estática seguiu os procedimentos da norma DIN (1982) e o ensaio de arrancamento de parafuso seguiu as indicações da ABNT (2013). Para a determinação da razão de compactação, tomou-se como base a massa específica do Pinus spp encontrada por Gorski et al. (2015) para o mesmo material $\left(410 \mathrm{~kg} \cdot \mathrm{m}^{-3}\right)$.

O modelo experimental utilizado para a análise estatística foi delineamento inteiramente casualizado (DIC). A distribuição da normalidade dos dados foi avaliada por meio do teste de Shapiro Wilk, enquanto a homogeneidade foi verificada pela aplicação do teste de Bartlett. Com os pressupostos atendidos, cada variável resposta foi submetida à análise de variância. Decorrendo rejeição da hipótese de nulidade, realizou-se o teste de médias de Scott-Knott a 95\% de probabilidade.

Quando não havia o atendimento aos pressupostos iniciais, os dados da propriedade de interesse eram transformados e posteriormente reavaliados. Ainda assim, permanecendo o não atendimento, era aplicada a estatística não paramétrica por meio do teste de Kruskal Wallis.

Além da análise estatística tradicional, sempre que possível, os valores médios de cada tratamento foram comparados com as normas de qualidade: ABNT (2013), ANSI (2009), CS (1968) e EN (2003).

\section{RESULTADOS E DISCUSSÃO}

\section{Propriedades físicas}

Os valores médios para massa específica, razão de compactação, espessura e teor de umidade dos painéis estão apresentados na Tabela 1, juntamente com os seus coeficientes de variação. Pode-se observar na tabela que o valor de massa específica estabelecido (650 kg.m³ $)$ não foi atingido em nenhum dos tratamentos, distanciando cada vez mais do nominal à medida que era aumentada a proporção de maravalhas, tanto que os tratamentos com $75 \%$ e 100\% desta matéria-prima foram estatisticamente diferentes dos demais.

Este fato é recorrente, uma vez que grande parte dos estudos com painéis de partículas / fibras apresentam diferença entre a massa específica nominal e a efetiva. Eleotério et al. (2000) cita que um dos fatores que pode contribuir para a diferença é o espalhamento das partículas / fibras durante a prensagem, determinando que o material se dispersasse por uma área maior que a planejada. Weber (2011) afirma que a diferença ocorre em função do retorno em espessura e a liberação de tensões das partículas após a prensagem dos painéis. Já Iwakiri et al. (2008), atribuem principalmente às perdas de materiais durante as fases de retirada das partículas da encoladeira, a formação do colchão e o carregamento da prensa. Enfim, pode-se dizer que a diferença está em função das condições opera- 
Cunha et al. - Efeito do teor de umidade da madeira na emissão de gases de efeito estufa no processo de carbonização

cionais em nível laboratorial, sem a automação e controle de precisão do processo industrial, ocasionando perdas de material durante o processo de manufatura dos painéis (IWAKIRI et al., 2012).

Tabela 1. Valores médios de massa específica, razão de compactação, teor de umidade e espessura.

Table 1. Medium values for specific gravity, compaction ratio, moisture content and thickness.

\begin{tabular}{|c|c|c|c|c|}
\hline Composição & M.E. $\left(\mathrm{kg} \cdot \mathrm{m}^{-3}\right)$ & Razão de compactação & Espessura (mm) & Teor de umidade (\%) \\
\hline $\mathrm{F}_{100 \%-\mathrm{M} 0 \%}$ & ${ }^{\mathrm{T} 1} 0,62_{(4,05)} \mathrm{a}$ & ${ }^{\mathrm{T} 1} 1,51_{(4,05)} \mathrm{a}$ & $\mathrm{T} 115,85_{(1,17)} \mathrm{a}$ & ${ }^{\mathrm{T} 19,76_{(4,33)} \mathrm{a}}$ \\
\hline$F_{75 \%-M 25 \%}$ & ${ }^{\mathrm{T} 2} 0,61_{(7,32)}^{\mathrm{a}}$ & $\mathrm{T} 21,50_{(7,32)} \mathrm{a}$ & $\mathrm{T} 215,95_{(1,34)}$ a & $\mathrm{T} 29,95_{(7,86)}^{(1,00)} \mathrm{a}$ \\
\hline $\mathrm{F}_{50 \%-\mathrm{M} 50 \%}$ & ${ }^{\mathrm{T} 3} 0,59_{(10,80)} \mathrm{a}$ & T31,46 ${ }_{(10,80)} \mathrm{a}$ & ${ }^{\mathrm{T}} 15,95_{(1,84)} \mathrm{a}$ & ${ }^{\mathrm{T}} 310,27_{(6,20)} \mathrm{a}$ \\
\hline$F_{25 \%-M 75 \%}$ & ${ }^{\mathrm{T} 4} 0,55_{(10,05)} \mathrm{b}$ & $\mathrm{T} 41,36_{(10,05)} \mathrm{b}$ & ${ }^{\mathrm{T} 4} 16,02_{(1,15)} \mathrm{a}$ & ${ }^{\mathrm{T}} 410,39_{(5,13)} \mathrm{a}$ \\
\hline $\mathrm{F}_{0 \%-\mathrm{M} 100 \%}$ & ${ }^{T 5} 0,55_{(10,38)} b$ & $T 51,36_{(10,38)} \mathrm{b}$ & ${ }^{T 5} 16,30_{(1,90)} \mathrm{b}$ & $\mathrm{T} 510,45_{(5,64)} \mathrm{a}$ \\
\hline Média & 0,58 & 1,44 & 16,01 & 10,16 \\
\hline
\end{tabular}

Legenda: F: fibra, M: maravalha, M.E.: massa específica, sobrescrito: tratamento, subscrito: coeficiente de variação (\%). Médias seguidas de mesma letra na coluna não diferem estatisticamente entre si.

Os maiores valores de massa específica obtidos para os tratamentos T1, T2, T3 (100\%, 75\% e $50 \%$ de fibras) em relação aos tratamentos T4, T5 (25\% e 0\% de fibras) pode ser atribuída a maior interação das fibras com o adesivo e também a melhor acomodação das fibras na estrutura do painel, o que reflete em menores perdas de processo, principalmente no menor espalhamento do material durante a prensagem e a menor liberação das tensões após a liberação da pressão na etapa de prensagem a quente dos painéis.

Quanto às normas de qualidade para classificação, verifica-se que os painéis podem ser definidos como de média massa específica, segundo a ABNT (2013). Tomando-se como referência a CS (1968), observa-se que somente os tratamentos T1 e T2 podem ser enquadrados como de média massa específica. Ainda, segundo especificações da ANSI (2009), nenhum tratamento se enquadrou como de média massa específica, em função de se encontrarem abaixo do valor mínimo de 640 $\mathrm{kg} \cdot \mathrm{m}^{-3}$ para tal classificação.

Como a razão de compactação é uma relação direta da massa específica do painel com a da espécie de madeira utilizada, a tendência foi a mesma da encontrada para a variável anterior, sendo que os valores apresentaram intervalo entre 1,36 no tratamento T5 e 1,51 no tratamento T1. Um ponto importante a ser destacado é a obtenção de valores dentro dos limites propostos por Maloney (1993) e Moslemi (1974), que é de 1,3 a 1,6 para que ocorra maior eficiência no processo de densificação e consolidação do painel até a sua espessura final. Assim, pode-se dizer que mesmo com a não obtenção da massa específica nominal dos painéis, obteve-se condições de densificação regulares de acordo com a literatura supracitada.

Para espessura final dos painéis $(15,55 \mathrm{~mm})$, verificou-se um acréscimo dimensional em todos os tratamentos, aumentando à medida que eram incorporadas as partículas de maravalha, sendo que o tratamento T5 foi diferenciado estatisticamente dos demais com espessura de $16,30 \mathrm{~mm}$.

Moslemi (1974) cita que o aumento da espessura dos painéis pode ser resultado da liberação parcial das tensões de compressão ocorrida logo após o final da prensagem na fase de produção do painel. O mesmo autor menciona ainda que quanto maior a altura do colchão a ser comprimido para uma mesma espessura final, maiores serão as tensões de compressão às quais o painel estará sujeito durante a operação de prensagem. No entanto, este fato não foi verificado no presente estudo, tendo em vista que o colchão com fibras era mais alto que o de maravalha; acredita-se que a maior interação entre fibras e adesivo, e a maior acomodação desta matéria-prima proporcionaram os menores valores de espessura final.

O valor médio de teor de umidade após a estabilização da massa foi de 10,16\%, sem diferença estatística entre os tratamentos. Salienta-se que os valores encontrados estão abaixo da umidade de equilíbrio da sala de climatização que é de $12 \%$. A diminuição da higroscopicidade dos painéis tem relação com a redução da madeira em partículas/fibras e a posterior incorporação de resina, parafina e outros aditivos (WU, 1999), e principalmente, às altas temperaturas aplicadas durante a etapa de prensagem, que fazem com que a pressão compacte as camadas externas do painel, tornando os sítios de adsorção menos disponíveis (DEL MENEZZI et al., 2007).

Ainda sobre o teor de umidade dos painéis, nota-se que os valores médios estão de acordo com o especificado pela ABNT (2013), que define intervalo entre de $5 \%$ a 11\%, e com o valor máximo da ANSI (2009) que é de $10 \%$. 
Na Tabela 2 estão apresentados os valores médios de absorção de água após 2 e 24 horas de imersão, bem como os valores de inchamento em espessura. Para absorção de água, denota-se que o tratamento T1 com $100 \%$ de fibras foi o que apresentou o menor valor médio, tanto após o primeiro ciclo de imersão $(12,73 \%)$, quanto após o segundo $(28,81 \%)$. Já, os demais tratamentos, foram significativamente diferentes do primeiro, de forma que com o aumento da porcentagem de maravalhas, os coeficientes também aumentaram, chegando a 44,88\% (2 h) e 81,87\% (24 h), ambos no tratamento T5 com 100\% de maravalhas em sua composição.

Tabela 2. Valores médios para absorção de água e inchamento em espessura.

Table 2. Medium values for the water absorption and thickness swelling.

\begin{tabular}{|c|c|c|c|c|}
\hline \multirow{2}{*}{ Composição } & \multicolumn{2}{|c|}{ A.A. (\%) } & \multicolumn{2}{|c|}{ I.E. (\%) } \\
\hline & 2 horas & 24 horas & 2 horas & 24 horas \\
\hline $\mathrm{F}_{100 \%}-\mathrm{M}_{0 \%}$ & $\mathrm{~T}_{1} 12,73_{(12,40)} \mathrm{a}$ & ${ }^{\mathrm{T} 1} 28,81_{(10,68)} \mathrm{a}$ & $\left.\mathrm{T}^{\mathrm{T}} 4,25_{(34,78}\right) \mathrm{a}$ & 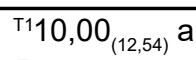 \\
\hline $\mathrm{F}_{75 \%}-\mathrm{M}_{25 \%}$ & T2 $22,78_{(9,36)} b$ & ${ }^{\mathrm{T}} 42,29_{(15,16)} \mathrm{b}$ & $\mathrm{T} 211,54_{(8,70)} \mathrm{b}$ & ${ }^{\mathrm{T}} 14,66_{(7,61)} \mathrm{b}$ \\
\hline $\mathrm{F}_{50 \%}-\mathrm{M}_{50 \%}$ & ${ }^{\mathrm{T}} 26,66_{(15,37)} \mathrm{b}$ & ${ }^{T} 352,54_{(14,11)} b$ & ${ }^{\mathrm{T}} 312,46_{(4,06)} \mathrm{b}$ & T315,82 $(4,97) b$ \\
\hline $\mathrm{F}_{25 \%}-\mathrm{M}_{75 \%}$ & ${ }^{\mathrm{T}} 35,59_{(12,51)} \mathrm{b}$ & ${ }^{\mathrm{T}} 64,73_{(10,57)} \mathrm{b}$ & ${ }^{\mathrm{T}} 413,75_{(6,97)} \mathrm{b}$ & ${ }^{\mathrm{T}} 17,21\left(_{7,70)} \mathrm{b}\right.$ \\
\hline$F_{0 \%}-M_{100 \%}$ & ${ }^{\mathrm{T}} 44,88_{(14.09)} \mathrm{C}$ & ${ }^{T 5} 81,87_{(1112)} \mathrm{b}$ & $\mathrm{T} 515,09_{(1676)} b$ & ${ }^{\mathrm{T} 5} 16,77_{(3519)} \mathrm{b}$ \\
\hline Média & 28,53 & 57,25 & 11,42 & 14,89 \\
\hline
\end{tabular}

Legenda: F: fibra, M: maravalha, A.A.: absorção de águas, I.E.: inchamento em espessura, sobrescrito: tratamento, subscrito: coeficiente de variação (\%). Médias seguidas de mesma letra na coluna não diferem estatisticamente entre si.

O fato do tratamento T1 ser composto por 100\% de fibras pode ter contribuído para este resultado em função da menor dimensão ter proporcionado um melhor recobrimento das fibras com resina, impedindo assim a entrada de água. Já o tratamento T5 pode ter sido prejudicado em função da menor disponibilidade de resina por unidade de área, o que tem como consequência uma menor adesão quando em contato com a água.

Os resultados encontrados no estudo para absorção de água estão próximos aos encontrados por França et al. (2016), que ao trabalhar com painéis homogêneos de fibras e partículas cepilhadas de Pinus spp com diferentes teores de resina, encontrou valores entre 5,45\% a 40,41\% para $2 \mathrm{~h}$, e entre $7,45 \%$ a $82,39 \%$ para $24 \mathrm{~h}$; abaixo dos valores encontrados por Brito et al. (2006), que ao trabalhar com painéis homogêneos de partículas provenientes de toretes e de maravalhas de Pinus elliottii Engelm, encontrou intervalo de valores de $47,90 \%$ a 54,16\% para 2 h e de $51,09 \%$ a 56,3\% para 24 h; e acima dos encontrados por Trianoski et al. (2011) que encontrou 7,94\% para 2 horas e 27,82\% para 24 horas em painéis de partículas homogêneos de Pinus taeda com 12\% de resina.

No inchamento em espessura, verifica-se que os valores médios seguiram a mesma tendência da absorção de água, onde o melhor tratamento foi o T1 com 100\% de fibras, sendo diferente estatisticamente dos demais que foram equivalentes tanto para $2 \mathrm{~h}$ quanto para $24 \mathrm{~h}$. Esta tendência também foi encontrada por França et al. (2016), que verificou que à medida que eram incorporadas partículas cepilhadas no painel, o inchamento em espessura aumentava de forma significativa, tanto para 2 h $(2,22 \%$ até $13,30 \%)$ quanto para 24 h (10,19\% até $23,40 \%)$.

Souza et al. (2012) estudou painéis aglomerados produzidos a partir de partículas de Pinus spp de origem laboratorial e industrial, no qual obteve para o industrial, valores médios de 3,99\% e $14,51 \%$ para 2 h e 24 h, respetivamente. Já para o laboratorial, 7,74\% para 2 h e 29,29\% para 24 h. Comparando com os valores médios do presente estudo, observa-se que para $2 \mathrm{~h}$ os valores encontrados foram superiores, enquanto que para $24 \mathrm{~h}$ foram equivalentes aos resultados obtidos nos painéis industriais e inferiores aos obtidos nos painéis laboratoriais.

Em relação ao atendimento aos critérios das normas de qualidade, verifica-se que quanto ao inchamento 24 horas, todos os tratamentos se enquadram nas normas CS (1968), ANSI (2009) e ABNT (2013) que estabelecem valores máximos de 35\%, 40\% e 18\% respectivamente. Já segundo a EN (2003) que estabelece 15\% como máximo, somente os tratamentos T1 e T2 atendem o pressuposto.

Com os resultados encontrados para as propriedades físicas dos painéis, pode-se dizer que o aumento da granulometria das partículas contribuiu negativamente com a massa específica nominal, razão de compactação, absorção de água e inchamento em espessura. 


\section{Propriedades mecânicas}

Os valores médios de MOE demonstram que os tratamentos T1 e T2 com 100\% e 75\% de fibras foram os que se destacaram estatisticamente dos demais com $1030 \mathrm{MPa}$ e $855 \mathrm{MPa}$, enquanto que para MOR somente o T1 obteve resultados satisfatórios que possibilitam a utilização dos painéis (Tabela 3).

Tabela 3. Valores médios para flexão estática.

Table 3. Medium values for static bending.

\begin{tabular}{|c|c|c|}
\hline \multirow{2}{*}{ Composição } & \multicolumn{2}{|c|}{ Flexão Estática (MPa) } \\
\hline & MOE & MOR \\
\hline $\begin{array}{l}\mathrm{F}_{100 \%}-\mathrm{M}_{0 \%} \\
\mathrm{~F}_{75 \%}-\mathrm{M}_{25 \%} \\
\mathrm{~F}_{50 \%}-\mathrm{M}_{50 \%} \\
\mathrm{~F}_{25 \%}-\mathrm{M}_{75 \%} \\
\mathrm{~F}_{0 \%}-\mathrm{M}_{100 \%} \\
\text { Média }\end{array}$ & $\begin{array}{cc}\mathrm{T} 11030_{(11,13)} & \mathrm{a} \\
\mathrm{T}^{\mathrm{T}} 855_{(16,48)} & \mathrm{a} \\
{ }^{\mathrm{T}} 739_{(15,00)} & \mathrm{b} \\
{ }^{\mathrm{T} 4} 640_{(15,10)} & \mathrm{b} \\
{ }^{\mathrm{T} 5} 521_{(19,14)} & \mathrm{b} \\
757 & \end{array}$ & $\begin{array}{cc}\mathrm{T} 124,66_{(12,21)} & \mathrm{a} \\
\mathrm{T} 216,67_{(18,03)} \mathrm{b} \\
\mathrm{T} 312,23_{(16,76)} \mathrm{C} \\
\mathrm{T} 47,90_{(18,13)} & d \\
{ }^{\mathrm{T}} 5,91_{(19,44)} & d \\
13,47 & \\
13,4\end{array}$ \\
\hline
\end{tabular}

Legenda: F: fibra, M: maravalha, MOE: Módulo de elasticidade, MOR: módulo de ruptura, sobrescrito: tratamento, subscrito: coeficiente de variação (\%). Médias seguidas de mesma letra na coluna não diferem estatisticamente entre si.

Brito et al. (2006) em seu estudo com painéis aglomerados de partículas e maravalhas de Pinus elliottii também encontraram a mesma tendência do presente estudo, onde a incorporação de maravalhas diminuiu os valores de MOE e MOR. No entanto, os valores encontrados pelo autor foram superiores, com intervalo de $1531 \mathrm{MPa}$ a $1067 \mathrm{MPa}$ para MOE e de 11,18 MPa a 8,04 MPa para MOR, sendo os valores inferiores para os painéis com 100\% de partículas proveniente da madeira maciça e superiores para os painéis com $100 \%$ de maravalhas.

Porém, outros autores encontraram outra tendência em relação à granulometria do material (partículas / fibras), como França et al. (2016) que observou que com o aumento da quantidade de partículas em relação a fibras no painel, os valores de MOE e MOR aumentaram. No entanto, cabe salientar que a autora utilizou partículas provenientes de processo industrial de produção de painéis, e não maravalhas. Fato semelhante ocorreu no estudo de Dacosta et al. (2005), que observou que com o aumento do uso de maravalhas houve um aumento do MOR e MOE, entretanto, a autora utilizou para este incremento, aumento na massa específica dos painéis e aumento na quantidade de adesivo.

Comparando os resultados de MOE e MOR com a ABNT (2013) que estabelece valor de rigidez e resistência mínimos de 1600 Mpa para MOE e 11 Mpa para MOR, verifica-se que todos os tratamentos ficaram abaixo do proposto para MOE, enquanto que para MOR, apenas os tratamentos T4 e T5 não atingiram o valor mínimo. Da mesma forma para a EN (2003), onde nenhum dos tratamentos atendeu os valores mínimos para o MOE (1600 MPa); já para MOR, apenas os tratamentos T1 e T2 foram superiores ao estabelecido (13 MPa) pela norma de referência.

Quanto à comparação com a ANSI (2009), nenhum dos tratamentos atingiram os valores mínimos para MOE, enquanto que para MOR, os tratamentos $\mathrm{T} 1$ e $\mathrm{T} 2$ foram enquadrados na classe M3-i e o T3 na classe MS.

Nos valores médios de adesão interna apresentados na Tabela 4, observa-se uma amplitude de variação de 0,25 MPa (T5) a 0,43 MPa (T1), onde os melhores tratamentos foram os compostos por $100 \%$ e $75 \%$ de fibras.

Tabela 4. Valores médios referentes à tração perpendicular.

Table 4. Medium values for the perpendicular traction.

\begin{tabular}{|c|c|}
\hline Composição & Adesão interna (MPa) \\
\hline $\begin{array}{l}\mathrm{F}_{100 \%}-\mathrm{M}_{0 \%} \\
\mathrm{~F}_{75 \%}-\mathrm{M}_{25 \%} \\
\mathrm{~F}_{50 \%}-\mathrm{M}_{50 \%} \\
\mathrm{~F}_{25 \%}-\mathrm{M}_{75 \%} \\
\mathrm{~F}_{0 \%}-\mathrm{M}_{100 \%} \\
\text { Média }\end{array}$ & $\begin{array}{c}{ }^{\mathrm{T} 1} 0,43_{(13,16)} \\
{ }^{\mathrm{T} 2} 0,36_{(17,78)} \\
{ }^{\mathrm{T} 3} 0,28_{(18,00)} \\
{ }^{\mathrm{T} 4} 0,30_{(12,47)} \\
{ }^{\mathrm{T} 5} 0,25_{(13,75)} \\
0 \\
0,32\end{array}$ \\
\hline
\end{tabular}

Legenda: F: fibra, M: maravalha, sobrescrito: tratamento, subscrito: coeficiente de variação (\%). Médias seguidas de mesma letra na coluna não diferem estatisticamente entre si. 
Em linhas gerais, segundo Fiorelli et al. (2015), pode-se dizer que a inclusão progressiva de partículas de maravalha nos painéis, proporciona uma diminuição sucessiva na adesão interna. Este fato é provavelmente devido à morfologia das partículas de maravalha que não conseguem preencher os vazios existentes do painel, prejudicando os resultados da propriedade avaliada.

Brito e Peixoto (2000) citam também que painéis feitos com partículas menores apresentaram uma maior resistência à tração perpendicular quando comparadas a partículas maiores, em função das partículas menores serem responsáveis por uma melhor uniformização do material e pela formação de menores espaços internos.

Comparando os resultados com a ABNT (2013) e a EN 312 (2003), que estabelecem valor mínimo de 0,35 MPa para a propriedade, nota-se que apenas os tratamentos T1 e T2 atenderam ao requisito. Quanto a CS (1968) que estipula 0,48 MPa, nenhum tratamento foi evidenciado. E ainda, em relação a ANSI (2009), que estabelece nas classes de qualidade, observa-se que o tratamento T1 pode ser classificados como M-2, o T2 como MS e os demais em nenhuma das classes fixadas pela norma.

$\mathrm{Na}$ resistência ao arrancamento do parafuso (Tabela 5), observa-se que os valores médios do arranchamento superficial $(1109,50 \mathrm{MPa})$ foram superiores ao de topo $(951,76 \mathrm{MPa})$, sendo novamente o tratamento que se destacou com os melhores resultados o T1 com 100\% de fibras. Já o tratamento T5, apresentou resultados $43 \%$ e $47 \%$ inferiores para superfície e topo, respectivamente.

Tabela 5. Valores médios para resistência ao arrancamento de parafuso.

Table 5. Medium values for the screw withdrawal resistance.

\begin{tabular}{|c|c|c|}
\hline \multirow{2}{*}{ Composição } & \multicolumn{2}{|c|}{ Arrancamento de Parafuso (N) } \\
\hline & Superfície & Topo \\
\hline $\mathrm{F}_{100 \%}-\mathrm{M}_{0 \%}$ & ${ }^{\mathrm{T} 1} 1481,83_{(18,78)} \mathrm{a}$ & ${ }^{\mathrm{T} 1} 1295,41_{(9,11)} \mathrm{a}$ \\
\hline $\mathrm{F}_{75 \%}-\mathrm{M}_{25 \%}$ & ${ }^{\mathrm{T}} 1089,33_{(38,13)} \mathrm{b}$ & ${ }^{\mathrm{T}} 2906,00_{(34,10)} \mathrm{b}$ \\
\hline $\mathrm{F}_{50 \%}-\mathrm{M}_{50 \%}$ & ${ }^{T} 31132,33_{(17,52)} b$ & ${ }^{\mathrm{T}} 991,83_{(28,60)} \mathrm{b}$ \\
\hline $\mathrm{F}_{25 \%}-\mathrm{M}_{75 \%}$ & ${ }^{\mathrm{T} 4} 1008,92_{(10,04)} \mathrm{b}$ & ${ }^{\mathrm{T}} 881,50_{(15,18)} \mathrm{b}$ \\
\hline$F_{0 \%}-M_{100 \%}$ & ${ }^{\mathrm{T}} 835,00_{(35,52)} \mathrm{C}$ & ${ }^{T 5} 684,08_{(54,05)} \mathrm{C}$ \\
\hline
\end{tabular}

Legenda: F: fibra, M: maravalha, sobrescrito: tratamento, subscrito: coeficiente de variação (\%). Médias seguidas de mesma letra na coluna não diferem estatisticamente entre si.

Trianoski et al. (2011) esclarece que em termos gerais e considerando a superfície e o topo, normalmente a superfície tende a apresentar os maiores resultados de resistência, devido principalmente ao gradiente de densidade formado durante o processo de prensagem e a localização do parafuso no ensaio de topo estar localizado exclusivamente no centro geométrico da espessura do painel, onde geralmente ocorre a menor densificação. A autora complementa que outro fato a ser discutido, refere-se à influência da razão de compactação que tende a reduzir os espaços vazios no painel e promover um maior contato entre as partículas, gerando assim uma maior resistência mecânica, conforme observado no arrancamento do parafuso de superfície.

Haselein et al. (2002) utilizoupartículas de Pinus elliottii com diferentes dimensões eobteve diferença estatística entre os tratamentos, onde os painéis formados por partículas de menor dimensão, forneceram os melhores resultados para a propriedade.

De acordo com a ABNT (2013), verifica-se que apenas os tratamentos T1, T2 e T3 atingiram o valor de $1020 \mathrm{MPa}$ para arrancamento de superfície, porém para o topo somente o T5 não atingiu o mínimo de 800N. Em relação a ANSI (2009), os painéis dos tratamentos T1, T2 e T3 podem ser classificados como M-3i e o tratamento T4 como M-S.

\section{CONCLUSÕES}

Os valores médios obtidos para massa específica dos painéis não atenderam ao nominal planejado, porém este fato não influenciou nos valores de razão de compactação.

Quanto à estabilidade dimensional dos painéis, proporcionado pelo inchamento em espessura, observou-se que todos os tratamentos apresentaram resultados regulares, principalmente àqueles com $100 \%$ e $75 \%$ de fibras, que atenderam a todas as normas de referência. 
Em relação às propriedades mecânicas avaliadas, o incremento da quantidade de maravalha nos painéis, ou reduziu os valores de módulo de elasticidade e adesão interna em decorrência da menor qualidade da adesão proporcionada pelas partículas de menor dimensão.

De forma geral, pode-se dizer que as composições analisadas no presente estudo são viáveis (para uma proporção máxima de $25 \%$ de maravalha).

\section{AGRADECIMENTOS}

A Madepar Indústria e Comércio de Madeiras LTDA, a Compensados Sudati Ltda, ao SENAI - Unidade Lages e a Fundação de Amparo à Pesquisa e Inovação do Estado de Santa Catarina (FAPESC).

\section{REFERÊNCIAS BIBLIOGRÁFICAS}

ABNT - Associação Brasileira de Normas Técnicas. NBR 14810: painéis de madeira de média densidade. Parte 2: requisitos e métodos de ensaio. Rio de Janeiro, 2013. 69p.

ABRAF - ASSOCIAÇÃO BRASILEIRA DE PRODUTORES DE FLORESTAS PLANTADAS. Anuário estatístico da ABRAF 2013: ano base 2013. Brasília: ABRAF, 2013. 146p.

ANSI - AMERICAN NATIONAL STANDARDS INSTITUTE. ANSI A 208.1: Mat formed wood particleboard: specifications. Gaithersburg: National Particleboards Association, 2009. 9p..

ABIMCI - ASSOCIAÇÃO BRASILEIRA DA INDÚSTRIA DE MADEIRA PROCESSADA MECANICAMENTE. Estudo setorial 2009, ano base 2008. Curitiba: ABIMCI, 2009.

ASTM - AMERICAN SOCIETY FOR TESTING AND MATERIALS - ASTM - D1037. Annual Book of ASTM Standards. Philadelphia: 2006.

BIAZUS, A.; HORA, A. B.; LEITE, B. G. P. Panorama de Mercado: Painéis de Madeira. BNDES Setorial, n. 32, p. 49-90. Rio de Janeiro, BNDES, 2010.

BRITO, E. O.; PEIXOTO, G. L. Avaliação da granulometria de partículas de Pinus taeda combinadas com adesivos comerciais para a fabricação de aglomerados. Revista Floresta e Ambiente. Rio de Janeiro, v. 7, n. 1, p. 60-67, jan./dez. 2000.

BRITO, E. O.; SÁ-ROCHA, J. D. ; VIDAURRE, G.B.; BATISTA, D. C.; PASSOS, P.R. de A.; MARQUES, L.G. da C.. Propriedades de chapas produzidas com resíduos do fruto de coco e partículas de pinus. Floresta e Ambiente, Seropédica, v.11, n.2, p. 01 - 06, 2004.

BRITO, E. O.; SAMPAIO, L. C.; OLIVEIRA, J. N.; BATISTA, D. C. Chapas de madeira aglomerada utilizando partículas oriundas de madeira maciça e de maravalhas. Scientia Forestalis, Piracicaba, n. 72, p. 17 - 21, 2006.

CAMPOS, C. I.; LAHR, F. A. R. Estudo comparativo dos resultados de ensaio de tração perpendicular para MDF produzido em laboratório com fibras de pinus e de eucalipto utilizando uréia formaldeído. Matéria, $\mathrm{v}$. 9, n. 1, p. 32-42, 2004.

COMMERCIAL STANDART. CS 236-66: Mat formed wood particleboard. Geneva, 1968. 18p.

DACOSTA, L. P. E.; HASELEIN, C. R.; SANTINI, E. J.; SCHNEIDER, P. R.; CALEGARI, L.. Qualidade das chapas de partículas aglomeradas fabricadas com resíduos do processamento mecânico da madeira de Pinus elliottii Engelm. Ciência Florestal, v. 15, n.3, p. 311-322, 2005.

DEL MENEZZI, C.H.S.; TOMASELLI, I.; SOUZA, M.R. Avaliação não-destrutiva de painéis de partículas orientadas (OSB) modificados termicamente. Parte 1: Efeito do tratamento térmico sobre a velocidade de propagação de ondas de tensão. Scientia Forestalis (IPEF), v. 35, p. 67-75, 2007.

DIN - DEUTSCHES INSTITUT FÜR NORMUNG. DIN 52362: Testing of wood chipboards bending test, determination of bending strength. Berlin, 1982. $40 \mathrm{p}$. 
ELEOTÉRIO, J.R.; TOMAZELLO FILHO, M; BORTOLETTO JÚNIOR, G. Propriedades físicas e mecânicas de painéis MDF de diferentes massas específicas e teores de resina. Ciência Florestal, v. 10, p. 75-90, 2000.

EN - EUROPPEAN STANDART. EN 312-2: Particleboards. Specifications. Berlin, 2003. 17p.

FIORELLI, J.; CHRISTOFORO, A.L.; LAHR, F.A.R.; NASCIMENTO, M.F.; CURTOLO, D.D.; SARTORI, L.; BELINI, U.L. Painéis de partículas monocamadas fabricados com resíduo de madeira e fibra de coco verde. Revista Scientia Forestalis, v. 43, n. 105, 2015.

FRANÇA, M.C.; CUNHA, A. B.; TRIANOSKI, R.; RIOS, P. D.; SCHIMALSKI, M. B. Produção de painéis aglomerados de fibras oversize de uma indústria de MDF. Scientia forestalis, v. 44, n. 111, 2016.

GORSKI, L.; CUNHA, A.B.; RIOS, P.D.; TRIANOSKI, R.; MARTARELLO, L.P. Produção de painéis de partículas orientadas (OSB) de Eucalyptus benthamii e Pinus spp. em diferentes combinações de camadas. Scientia Forestalis (IPEF), v. 43, p. $367-375,2015$.

HASELEIN, C. R.; CALEGARI, L.; BARROS, M. V.; HACK, C.; HILLIG, E.; PAULESKI, D. T.; POZZERA, F. Resistência mecânica e à umidade de painéis aglomerados com partículas de madeira de diferentes dimensões. Ciência Florestal, Santa Maria, v. 12, n. 2, p. 127 - 134. 2002.

HRÁZSKÝ, J.; KRÁL, P. The influence of particle composition in a three-layer particleboard on its physical and mechanical properties. Journal of Forest Sceince, v.49, n.2, p.83 - 93, 2003.

IWAKIRI, S. Painéis de Madeira Reconstituída. Curitiba: FUPEF,2005, p.247.

IWAKIRI, S.; STINGHEN, A. B. M.; SILVEIRA, E. L. da; ZAMARIAN, E. H. C; PRATA, J. G; BRONOSKI, M. Influência da massa específica sobre as propriedades mecânicas de painéis aglomerados. Floresta, Curitiba, v.38, n.3, p. 487 - 493, 2008.

IWAKIRI, S.; MATOS, J. L. M. DE; TRIANOSKI, R.; PRATA, J. G. Produção de painéis aglomerados homogêneos e multicamadas de Melia azedarach (CINAMOMO) e Pinus taeda com diferentes teores de resina. Cerne, Lavras, v. 18, n. 3, p. 465 - 470, 2012.

MALONEY, T. M. Modern particleboard and dry-process fiberboard manufacturing. San Francisco: M. Freeman, 1993. 689 p.

MIGUEL, A. M.; DANIELLI, D.; RIOS, P. D.; VIEIRA, H. C.; KNIESS, D. D. C. Controle da qualidade das toras no pátio e dos painéis mdf. Anais do XV Encontro Brasileiro em Madeiras e em Estruturas de Madeira - XV EBRAMEM. Curitiba, 2016.

MOSLEMI, A. A. Particleboard: materials. London: Southern Illinois University, 1974. v. 1, 244 p.

SOUZA, K. B.; ALMEIDA, K. N. S.; GUIMARÃES JUNIOR, J. B.; GUIMARÃES NETO, R. M. Comparação das propriedades físicas de painéis aglomerados de Pinus de origem industrial e laboratorial. Scientia Plena. Bom Jesus, v.8, p. 1-5, 2012.

TRIANOSKI, R.; IWAKIRI, S.; MATOS, J.L.M.; PRATA, J.G. Avaliação de espécies alternativas de rápido crescimento para a produção de painéis de madeira aglomerada de três camadas. Scientia Forestalis (IPEF), v. 39, p. 97-104, 2011.

WEBER, C. Estudo sobre viabilidade de uso de resíduos de compensados, MDF e MDP para produção de painéis aglomerados. 2011. 87 p. Dissertação (Mestrado em Ciências Florestais) - Universidade Federal do Paraná, Curitiba.

WU, Q. Application of Nelson's sorption isotherm to wood composites and overlays. Wood and Fiber Science, v.28, n.2, p. 227 - 239, 1999.

Recebido em 26/05/2015

Aceito para publicação em 19/04/2016

Sci. For., Piracicaba, v. 44, n. 112, p. 841-849, dez. 2016 DOI: dx.doi.org/10.18671/scifor.v44n112.06 
